\title{
ENDOMORPHISM RINGS OF PROJECTIVE MODULES
}

\author{
BY \\ ROGER WARE
}

\begin{abstract}
The object of this paper is to study the relationship between certain projective modules and their endomorphism rings. Specifically, the basic problem is to describe the projective modules whose endomorphism rings are (von Neumann) regular, local semiperfect, or left perfect.

Call a projective module regular if every cyclic submodule is a direct summand. Thus a ring is a regular module if it is a regular ring. It is shown that many other equivalent "regularity" conditions characterize regular modules. (For example, every homomorphic image is flat.) Every projective module over a regular ring is regular and a number of examples of regular modules over nonregular rings are given. A structure theorem is obtained: every regular module is isomorphic to a direct sum of principal left ideals. It is shown that the endomorphism ring of a finitely generated regular module is a regular ring. Conversely, over a commutative ring a projective module having a regular endomorphism ring is a regular module. Examples are produced to show that these results are the best possible in the sense that the hypotheses of finite generation and commutativity are needed. An application of these investigations is that a ring $R$ is semisimple with minimum condition if and only if the ring of infinite row matrices over $R$ is a regular ring.

Next projective modules having local, semiperfect and left perfect endomorphism rings are studied. It is shown that a projective module has a local endomorphism ring if and only if it is a cyclic module with a unique maximal ideal. More generally, a projective module has a semiperfect endomorphism ring if and only if it is a finite direct sum of modules each of which has a local endomorphism ring.
\end{abstract}

1. Preliminaries. Throughout this paper, unless otherwise indicated, all modules over a ring $R$ will be understood to be left $R$-modules. $R$ will always have a unit, and every module will be unitary. All homomorphisms of $R$-modules will be written on the right so that if $M$ is an $R$-module and $S=\operatorname{Hom}_{R}(M, M)$ then $M$ becomes an $R-S$ bimodule, sometimes written ${ }_{R} M_{S}$. For all notions of homological algebra the reader is referred to [3]. This section consists of definitions, notation terminology, and basic facts about projective modules which will be used in the later ones.

Let $R$ be a ring, $M$ an $R$-module, and $N$ a submodule of $M$. We say $N$ is small in $M$ if whenever $K$ is a submodule of $M$ with $N+K=M$ then $K=M$. Dually, $N$ is large in $M$ if $N \cap K=0$ always implies $K=0$.

Received by the editors July 10, 1970.

AMS 1969 subject classifications. Primary 1640, 1656, 1690; Secondary 1820.

Key words and phrases. Projective module, endomorphism ring, Jacobson radical, von Neumann regular ring, local ring, semiperfect ring, left perfect ring, left $T$-nilpotent, flat module, semiprime ring, row finite matrices, injective module.

Copyright (C) 1971, American Mathematical Society 
If $M$ is an $R$-module, the radical of $M$, denoted $J(M)$, is defined to be the intersection of all maximal submodules of $M$. It may happen that $M$ has no maximal submodules in which case $J(M)=M$. Thus, for a ring $R, J(R)$ is the Jacobson radical of $R$. It is an easy exercise to show that, for any module $M, J(M)$ coincides with the sum of all small submodules of $M$.

If $P$ is a projective $R$-module then $P$ is a direct summand of a free module and hence $J(P)=J(R) P$. Bass proved [1, Proposition 2.7] that if $P \neq 0$ is a projective module then $J(R) P \neq P$. Thus every projective module has a maximal submodule.

The relationship between the radical of a projective module and the Jacobson radical of its endomorphism ring is contained in the following proposition:

Proposition 1.1. Let $P$ be a projective $R$-module, $S=\operatorname{Hom}_{R}(P, P)$ the ring of endomorphisms of $P$, and $T=\{f \in S \mid \operatorname{Im} f$ is small in $P\}$. Then

(1) $J(S)=T$.

(2) $J(S) \subset \operatorname{Hom}_{R}(P, J(P))$.

(3) There is a ring epimorphism $\Phi: S \rightarrow \operatorname{Hom}_{R}(P / J(P), P / J(P))$ with

$$
\operatorname{Ker} \Phi=\operatorname{Hom}_{R}(P, J(P)) \text {. }
$$

(4) If $J(P)$ is small in $P$ then $J(S)=\operatorname{Hom}_{R}(P, J(P))$ and hence

$$
S / J(S) \cong \operatorname{Hom}_{R}(P / J(P), P / J(P)) \text {. }
$$

Proof. (1) The proof that $T \subset J(S)$ is contained in the proof of Theorem 2.4 in [8] and will be omitted here.

Now let $f \in J(S)$ and suppose $\operatorname{Im} f+K=P$. Let $\pi: P \rightarrow P / K$ be the natural map. Then $\operatorname{Im} f+K=P$ implies that $f \pi: P \rightarrow P / K$ is an epimorphism so since $P$ is projective there is an $R$-homomorphism $g: P \rightarrow P$ making the following diagram commutative:

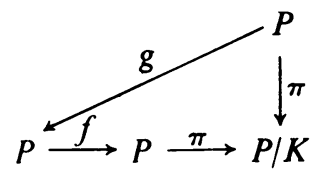

Then $x-x g f \in K$ for all $x \in P$, i.e. $\operatorname{Im}(1-g f) \subset K$. But $f \in J(S)$ implies $1-g f$ is an isomorphism on $P$. Thus $K=\operatorname{Im}(1-g f)=P$. Therefore $\operatorname{Im} f$ is small, so $f \in T$.

(2) By an earlier remark $J(P)$ must contain all small submodules of $P$, so if $f \in J(S)$ then $\operatorname{Im} f \subset J(P)$ by (1) and hence $f \in \operatorname{Hom}_{R}(P, J(P))$.

(3) $\Phi$ is defined as follows: Let $f \in S$. Since $J(P)=J(R) P, J(P) f \subset J(P)$. Therefore there is a unique map $\bar{f}$ making the following diagram commutative:

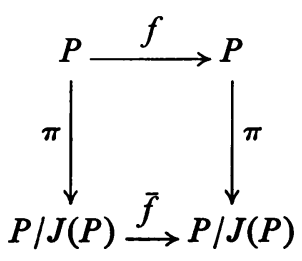


(where $\pi$ is natural). Define $\Phi(f)=\bar{f}$. Then $\Phi$ is easily seen to be a ring homomorphism of $S$ into $\operatorname{Hom}_{R}(P / J(P), P / J(P))$. Moreover, the projectivity of $P$ guarantees that $\Phi$ is an epimorphism. Finally, $f \in \operatorname{Ker} \Phi$ if and only if $\bar{f}=0$ on $P / J(P)$ if and only if $\operatorname{Im} f \subset J(P)$ if and only if $f \in \operatorname{Hom}_{R}(P, J(P))$.

(4) This is immediate from (1), (2), and (3).

REMARK. There are numerous examples which show that in general $J(S) \neq \operatorname{Hom}_{R}(P, J(P))$. However, if $P$ is a finitely generated projective then by Nakayama's lemma, $J(P)=J(R) P$ is small in $P$ so we have equality. This fact will be used in later portions of this paper.

Let $M$ be an $R$-module. A projective cover of $M$ is an epimorphism $P \rightarrow M$ with small kernel, where $P$ is projective. Dually, an injective envelope of $M$ is an injective module $E$ containing $M$ with $M$ large in $E$. While it is known (see [5]) that every module $M$ has a unique (up to an isomorphism fixing $M$ ) injective envelope, a module will not in general possess a projective cover. For example an abelian group $A$ has a projective cover if and only if $A$ is free. Concerning the uniqueness of projective covers (when they exist), Bass [1, Lemma 2.3] has shown:

("Uniqueness of Projective Covers".) Suppose $P \rightarrow M \rightarrow 0$ is a projective cover and $0 \rightarrow K \rightarrow P^{\prime} \rightarrow M \rightarrow 0$ exact with $P^{\prime}$ projective. Then $P^{\prime}=P_{1} \oplus P_{2}$ with $P_{1} \cong P, P_{2} \subset K$, and $P_{1} \cap K$ small in $P_{1}$.

Thus if $P$ is a projective cover of $M$ then $P$ is unique up to isomorphism. If $M$ has a projective cover we will denote it by $P(M)$. The injective envelope of $M$ will be denoted by $E(M)$.

In [1], Bass defines a ring $R$ to be left perfect if every left $R$-module has a projective cover and defines $R$ to be semiperfect if every cyclic (left) $R$-module has a projective cover. He shows that

(1) $R$ is left perfect if and only if $J(R)$ is left $T$-nilpotent and $R / J(R)$ has minimum condition.

Recall that an ideal $N$ in a ring $R$ is left $T$-nilpotent iff for any sequence $\left\{a_{i}\right\}_{i=1}^{\infty} \subset N$ there exists $n$ such that $a_{1} a_{2} \cdots a_{n}=0$.

(2) $R$ is semiperfect if and only if $R / J(R)$ has minimum condition and idempotents can be lifted modulo $J(R)$.

For these and other facts about projective covers, perfect rings, and semiperfect rings the reader is referred to [1].

The next two elementary facts about projective modules will be used extensively in what follows.

(1) ("Dual Basis Lemma" [3, VII, Proposition 3.1].) An $R$-module $P$ is projective if and only if there exist subsets $\left\{x_{i}\right\}$ of $P$ and $\left\{f_{i}\right\}$ of $\operatorname{Hom}_{R}(P, R)$ such that for each $x \in P, x f_{i}=0$ for almost all $i$, and $x=\sum_{i}(x) f_{i} x_{i}$. Moreover, if $P$ is projective, $\left\{x_{i}\right\}$ may be taken to be any generating set of $P$.

Let $M$ be an $R$-module and $T$ a subset of $M$. Then we define

$$
\mathrm{Ann}_{R} T=\{a \in R \mid a T=0\}
$$


Clearly, $\mathrm{Ann}_{R} T$ is a left ideal of $R$. It is also clear that $\mathrm{Ann}_{R} M$ is a two-sided ideal of $R$.

(2) $P=R x$ is a cyclic projective if and only if $A_{n} n_{R} x$ is a direct summand of $R$ if and only if $P \cong R e$ where $e^{2}=e \in R$.

Notation. If $R$ is a ring we denote by ${ }_{R} \mathscr{M}$ the category of left $R$-modules. For any index set $I$ and any $R$-module $M$ we let $M^{(I)}=\sum_{i \in I} \oplus M_{i}$, where $M_{i}=M, i \in I$. If $n$ is a positive integer then

$$
M^{n}=M^{(I)} \quad \text { where } I=\{1, \ldots, n\} .
$$

Thus $R^{n}$ will denote the standard free $R$-module of rank $n$.

We say that an $R$-module $M$ is a generator for ${ }_{R} \mathscr{M}$ if there exists a set $I$ such that $R$ is a homomorphic image of $M^{(I)}$. This is the same as saying that every $R$-module can be realized as a homomorphic image of a direct sum of copies of $M$. It is clear that $M$ is a generator for ${ }_{R} \mathscr{M}$ if and only if $\sum_{f \in \mathrm{Hom}_{R}(M, R)} M f=R$.

Proposition 1.2. Let $R$ be a ring and $P$ a projective $R$-module. Then $P$ is a generator for ${ }_{R} \mathscr{M}$ if and only if every simple $R$-module is a homomorphic image of $P$.

Proof. If $P$ is a generator then there is a set $I$ and an exact sequence $P^{(I)} \stackrel{f}{\rightarrow} R \rightarrow 0$. Thus if $S$ is a simple $R$-module we have an epimorphism $g: P^{(I)} \rightarrow S$. Now $P^{(I)}=\sum_{i \in I} \oplus P_{i}, P_{i}=P, i \in I$. Let $g_{i}=g \mid P_{i}$.

If $g_{i}=0$ for all $i$ then $g=0$. Hence $g \neq 0$ implies there exists $i \in I$ such that $g_{i}: P \rightarrow S$ is a nonzero homomorphism.

Conversely, suppose every simple module can be realized as a homomorphic image of $P$. Let $T=\sum_{f \in \mathrm{Hom}_{R}(P, R)} P f$. If $T=R$ then $P$ is a generator. If $T \neq R$ then $T$ can be embedded in a maximal left ideal $L$ of $R$. Since $R / L$ is simple there exists an epimorphism $g: P \rightarrow R / L$ and since $P$ is projective there is a linear map $f: P \rightarrow R$ such that the following diagram is commutative:

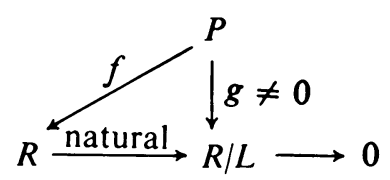

Therefore $P f \notin L$, but $P f \subset T$, a contradiction.

We say an $R$-module $P$ is a progenerator for ${ }_{R} \mathscr{M}$ if $P$ is a finitely generated projective generator in ${ }_{R} \mathscr{M}$. An $R$-module $M$ is said to be faithful if $\mathrm{Ann}_{R} M=0$. Note that every module $M$ is faithful over the ring $\bar{R}=R / \mathrm{Ann}_{R} M$.

Proposition 1.3. Let $R$ be a commutative ring and $P$ a finitely generated projective $R$-module. Then $P$ is a progenerator if and only if $P$ is faithful.

REMARK. This proposition is well known but as its proof is elementary we include it here for the convenience of the reader:

If $a \in \mathrm{Ann}_{R} P$ then $a P^{(I)}=0$ for any set $I$. Hence if $P$ is a generator then $a R=0$; i.e. $a=0$. Thus $P$ is faithful. 
Conversely, suppose $P$ is a finitely generated faithful projective $R$-module. Then by the dual basis lemma, if $x_{1}, \ldots, x_{n}$ generate $P$ there exist $f_{1}, \ldots, f_{n} \in \operatorname{Hom}_{R}(P, R)$ such that for each $x \in P$

$$
x=\sum_{j=1}^{n}(x) f_{j} x_{j} .
$$

Therefore, for each $i=1, \ldots, n$,

$$
x_{i}=\sum_{j=1}^{n}\left(x_{i}\right) f_{j} x_{j} .
$$

Hence for all $i=1, \ldots, n$,

$$
\sum_{j=1}^{n}\left(x_{i} f_{j}-\delta_{i j}\right) x_{j}=0
$$

Let $A$ be the matrix $\left(x_{i} f_{j}-\delta_{i j}\right), i, j=1, \ldots, n$, and let $\Delta=\operatorname{det} A$. (The commutativity of $R$ guarantees the existence of $\Delta$.) Then $\Delta=(-1)^{n}+a$ where

$$
a \in T=\sum_{f \in \operatorname{Hom}_{R}(P, R)} P f .
$$

But $\left(^{*}\right)$ implies that $\Delta x_{j}=0$ for all $j=1, \ldots, n$, so since $R$ is commutative, $\Delta P=0$. But $P$ is faithful so we must have $\Delta=0$. Therefore $(-1)^{n}=-a \in T$ so $1 \in T$, i.e. $T=R$ and $P$ is a generator. Since $P$ is already projective and finitely generated, $P$ is a progenerator.

2. Regular modules. A ring $R$ is (von Neumann) regular if for every $a \in R$ there exists an element $b \in R$ such that $a b a=a$.

It is well known that for a ring $R$ the following statements are equivalent:

(1) $R$ is regular.

(2) Every cyclic $R$-module is flat.

(3) Every principal left ideal is a direct summand.

(4) Every finitely generated left ideal is a direct summand.

(5) For every left ideal $K$ and every right ideal $I, I \cap K=I K$.

One notes that conditions (2)-(5) are module theoretic and the following proposition shows that their equivalence is essentially dependent on the fact that ${ }_{R} R$ is a projective $R$-module:

Proposition 2.1. Let $R$ be a ring and $P$ a projective $R$-module. Then the following statements are equivalent:

(1) Every homomorphic image of $P$ is flat.

(2) Every cyclic submodule of $P$ is a direct summand.

(3) Every finitely generated submodule of $P$ is a direct summand.

(4) For every submodule $K$ of $P$ and every right ideal $I$ of $R, I P \cap K=I K$.

The proof of Proposition 2.1 makes use of the following lemma: 
LEMMA 2.2. Let $R$ be $a$ ring and $0 \rightarrow K \rightarrow P \rightarrow M \rightarrow 0$ an exact sequence of $R$ modules with $P$ projective. Then the following statements are equivalent:

(1) $M$ is flat.

(2) Given any $x \in K$, there exists a homomorphism $g: P \rightarrow K$ such that $x g=x$.

(3) Given any $x_{1}, \ldots, x_{n}$ in $K$ there exists a homomorphism $g: P \rightarrow K$ such that $x_{i} g=x_{i}$ for $i=1, \ldots, n$.

Proof. Chase [4, Proposition 2.1] proved the lemma when $P$ is a free module. Thus choose $F$ free such that $F=P \oplus Q$. Then $F / K \cong P / K \oplus Q \cong M \oplus Q$ so we have an exact sequence

$$
0 \rightarrow K \rightarrow F \rightarrow M \oplus Q \rightarrow 0 .
$$

Since $Q$ is projective $M \oplus Q$ is flat iff $M$ is flat.

(1) $\Rightarrow$ (3). Let $x_{1}, \ldots, x_{n} \in K$ and suppose $M$ is flat. Then $M \oplus Q$ is flat so there exists $g^{\prime}: F \rightarrow K$ such that $x_{i} g^{\prime}=x_{i}, i=1, \ldots, n$. Let $g=\left.g^{\prime}\right|_{P}$. Then $g: P \rightarrow K$ and $x_{i} \in P$, so $x_{i} g=x_{i}, i=1, \ldots, n$.

(3) $\Rightarrow$ (2). Obvious.

(2) $\Rightarrow(1)$. Assume (2) and let $x \in K$. Then there exists $g: P \rightarrow K$ such that $x g=x$. Extend $g$ to $g^{\prime}: F \rightarrow K$ by defining $y g^{\prime}=0$ for $y \in Q$. Then $x g^{\prime}=x$ so by Chase's result applied to $\left(^{*}\right), M \oplus Q$ is flat. Therefore $M$ is flat.

Proof of Posposition 2.1. (1) $\Leftrightarrow$ (4). Let $0 \rightarrow K \rightarrow P \rightarrow M \rightarrow 0$ be exact. By [3, p. 122, Exercise 5], $M$ is flat iff for every right ideal $I$ of $R, I P \cap K=I K$.

(1) $\Rightarrow$ (3). Suppose $K=R x_{1}+\cdots+R x_{n}$ is a finitely generated submodule of $P$. Then we have an exact sequence

$$
0 \rightarrow K \rightarrow P \rightarrow P / K \rightarrow 0
$$

and by (1), $P / K$ is flat. Thus by the lemma there exists an $R$-homomorphism $g: P \rightarrow K$ such that $x_{i} g=x_{i}, i=1, \ldots, n$; i.e. $K$ is a direct summand of $P$.

(3) $\Rightarrow$ (2). Obvious.

(2) $\Rightarrow$ (1). Let $0 \rightarrow K \rightarrow P \rightarrow M \rightarrow 0$ be exact. Then for each $x \in K, R x$ is a direct summand of $P$ so there exists a homomorphism $g: P \rightarrow K$ such that $x g=x$. By Lemma 2.2, this implies that $M$ is flat.

Definition 2.3. We call a projective module satisfying the equivalent conditions of Proposition 2.1 a regular module.

EXAMPLES OF REgUlAR MODUles. (1) Any projective module over a regular ring is a regular module.

(2) Any semisimple projective module is a regular module. (By a semisimple module we mean one which is a (direct) sum of simple submodules.)

Recall that a ring is semiprime if it has no nonzero nilpotent (left) ideals. Thus the socle (=sum of minimal left ideals) of a semiprime ring will be a regular module. Note that since a minimal left ideal of a semiprime ring is a direct summand it will be projective. 
(3) It is known, cf. [7, Theorem 23, p. 112], that every ring $R$ possesses a unique largest two-sided ideal $M$ with respect to the property that for each $a \in M$ there exists $b \in M$ with $a b a=a$.

If $R$ is left noetherian or left perfect then $M$ is a regular (left) $R$-module.

Proof. First note that if $a \in M$ then $R=R a \oplus R(1-b a)$ so $R a$ is a direct summand of $R$, hence of $M$. Thus we need only show that $M$ is projective.

We assert that every finitely generated $R$-submodule of $M$ is cyclic.

The proof of this is a straightforward computation -in fact, identical to showing that in a regular ring every finitely generated left ideal is principal-and will be omitted here.

If $R$ is left noetherian this implies that $M=R a, a \in R$. But $R a$ is a direct summand of $R$, hence projective, i.e. $M$ is projective.

In any event, $M$ is the direct limit (union) of its finitely generated submodules, each of which will be cyclic and hence projective. But over a left perfect ring a direct limit of projective modules is again projective [1, Theorem P] so if $R$ is left perfect then $M$ is projective.

(4) Let $R$ be a regular ring and $F$ an infinitely generated free $R$-module. Let $S=\operatorname{Hom}_{R}(F, F)$. Choose a basis $\left\{x_{i}\right\}_{i \in I}$ for $F$ and write $F=\sum \oplus R x_{i}$. For each $i \in I$, let $p_{i}: F \rightarrow R x_{i}$ be the projection onto $R x_{i}$. Let $P$ be the right ideal of $S$ generated by the $p_{i}$. Since the $p_{i}$ are orthogonal idempotents of $S$ it is easy to see that $P=\sum_{i \in I} \oplus p_{i} S$ (as a right $S$-module) and hence $P$ is a projective (right) $S$-module.

We will see later (following Theorem $3.6, \S 3$ ) that $P$ is a regular right $S$-module but that $S$ is not in general a regular ring. In fact, $S$ will be regular if and only if $R$ has the minimum condition. The reader will note that when $R$ is a field, $P$ is the ideal of endomorphisms of finite rank.

Properties of regular modules. (1) Let $\left\{P_{i}\right\}_{i \in I}$ be a family of projective modules. Then $\sum_{i} \oplus P_{i}$ is regular if and only if each $P_{i}$ is regular.

Proof. Suppose $\sum_{i} \oplus P_{i}$ is regular. Then for each $i \in I$ every homomorphic image of $P_{i}$ is also a homomorphic image of $\sum \oplus P_{i}$, hence is flat. Therefore each $P_{i}$ is regular.

For the converse we need a lemma, the proof of which is standard and will be omitted.

LeMmA 2.4. Let $0 \rightarrow A \rightarrow B \rightarrow C \rightarrow 0$ be an exact sequence of left $R$-modules.

(1) If $B$ and $C$ are flat then $A$ is flat.

(2) If $A$ and $C$ are flat then $B$ is flat.

Now suppose $P_{1}, P_{2}$ are regular modules and let $P_{1} \oplus P_{2} \stackrel{f}{\rightarrow} M \rightarrow 0$ be exact. Then $M=P_{1} f+P_{2} f$ and we have exact sequences

(a) $0 \rightarrow P_{1} f \cap P_{2} f \rightarrow M \rightarrow P_{1} f / P_{1} f \cap P_{2} f \oplus P_{2} f / P_{1} f \cap P_{2} f \rightarrow 0$;

(b) $0 \rightarrow P_{1} f \cap P_{2} f \rightarrow P_{1} f \rightarrow P_{1} f / P_{1} f \cap P_{2} f \rightarrow 0$. 
Now $P_{1}, P_{2}$ regular implies that $P_{1} f, P_{2} f, P_{i} f / P_{1} f \cap P_{2} f, i=1,2$, are flat. Thus $P_{1} f / P_{1} f \cap P_{2} f \oplus P_{2} f / P_{1} f \cap P_{2} f$ is flat. Therefore, by Lemma 2.4, applied to (b), $P_{1} f \cap P_{2} f$ is flat, and now applied to (a), $M$ is flat. Thus $P_{1} \oplus P_{2}$ is regular. By induction it follows that any finite direct sum of regular modules is regular.

Now suppose $\left\{P_{i}\right\}_{i \in I}$ is any family of regular modules and let $P=\sum_{i \in I} \oplus P_{i}$. Let $x \in P$. Then $x=x_{i_{1}}+\cdots+x_{i_{n}}, x_{i_{j}} \in P_{i_{j}}, j=1, \ldots, n$. Then $x \in P_{i_{1}} \oplus \cdots \oplus P_{i_{n}}$. But $P_{i_{1}} \oplus \cdots \oplus P_{i_{n}}$ is a regular module so $R x$ is a direct summand of it. Therefore $R x$ is a direct summand of $P$. Hence $P$ is regular.

As a consequence of Lemma 2.4 we have

(2) Every submodule of a regular module is flat.

(3) If $P$ is a regular module then $J(P)=0$.

Proof. Since every cyclic submodule of $P$ is a direct summand, $P$ can have no nonzero small submodules, i.e. $J(P)=0$.

As a consequence of (3) and the fact that $J(P)=J(R) P$ for projective modules we have

(4) If $R$ is a ring possessing a faithful regular projective then $J(R)=0$. Thus if $R$ is any ring and $P$ is a regular $R$-module then $A n_{R} P$ is an intersection of maximal, left ideals.

For any (left) $R$-module $M$, the singular submodule of $M$ is

$$
Z_{R}(M)=\left\{x \in M \mid \mathrm{Ann}_{R} x \text { is a large left ideal of } R\right\} .
$$

(5) If $P$ is a regular module then $Z_{R}(P)=0$.

Proof. If $x \in P$ then $R x$ is a direct summand of $P$, hence projective. Therefore $\operatorname{Ann}_{R} x$ is a direct summand of $R$ and thus will not be large unless $x=0$.

A result, originally due to Kaplansky [11, Theorem 6], says that if $R$ is commutative then $R$ is a regular ring if and only if every simple $R$-module is injective. For regular modules we have

Proposition 2.5. Suppose $R$ is a commutative ring and $P$ is a regular $R$-module. Then every simple homomorphic image of $P$ is injective.

The proof of Proposition 2.5 requires a lemma.

LEMma 2.6. Suppose $R$ is a commutative ring and $S$ is a simple $R$-module. Then $S$ is flat if and only if $S$ is injective.

Proof. Let $E$ be the injective envelope of the direct sum of one copy of each of the simple $R$-modules. Thus $E=E\left(\sum_{i \in I} \oplus S_{i}\right)$ where $\left\{S_{i}\right\}_{i \in I}$ is the family of all (isomorphism types) of simple $R$-modules and if $i \neq j$ then $S_{i} \not S_{j}$. Then it is easy to see that for any $R$-module $M, \operatorname{Hom}_{R}(M, E)=0$ if and only if $M=0$. We assert that if $S \subset E$ is simple then $S=S_{i_{0}}$ for some $i_{0} \in I$.

$\sum_{i \in I} \oplus S_{i}$ is a large submodule of $E$ so $S \cap\left(\sum_{i} \oplus S_{i}\right) \neq 0$. Since $S$ is simple this implies there exist indices $i_{1}, \ldots, i_{n} \in I$ such that $S \subset S_{i_{1}} \oplus \cdots \oplus S_{i_{n}}$. Let $0 \neq x \in S$. Then $S=R x$ and $x=x_{i_{1}}+\cdots+x_{i_{n}}, x_{i_{i}} \in \dot{S_{i_{j}}}$ and not all $x_{i,}=0$. If $a x=0, a \in R$, then 
$a x_{i_{1}}+\cdots+a x_{i_{n}}=0$. Then $a x_{i_{k}} \in\left(\sum_{j \neq k} \oplus S_{i_{1}}\right) \cap S_{i_{k}}$ and hence $a x_{i_{k}}=0, k=1, \ldots, n$. Therefore $\mathrm{Ann}_{R} x \subset \mathrm{Ann}_{R} x_{i_{k}}, k=1, \ldots, n$. Since $S$ is simple, $\mathrm{Ann}_{R} x$ is a maximal ideal of $R$ so for each $k$, either $\mathrm{Ann}_{R} x_{i_{k}}=R$ or $\mathrm{Ann}_{R} x_{i_{k}}=\mathrm{Ann}_{R} x$. Since the simple modules $S_{i_{1}}, \ldots, S_{i_{n}}$ are nonisomorphic this implies there exists $k_{0}$ such that $\operatorname{Ann}_{R} x=\operatorname{Ann}_{R} x_{i_{k_{0}}}$ and $\mathrm{Ann}_{R} x_{i_{k}}=R, k \neq k_{0}$, i.e. $x_{i_{k}}=0, k \neq k_{0}$ and $S=S_{i_{k_{0}}}$, proving the assertion.

Now let $S$ be any simple $R$-module and let $S_{i_{0}}$ be the copy of $S$ in $E$. Then $\operatorname{Hom}_{R}\left(S, S_{i_{0}}\right) \subset \operatorname{Hom}_{R}(S, E)$ and if $0 \neq f \in \operatorname{Hom}_{R}(S, E)$ then $S \cong \operatorname{Im} f \subset E$ so by the above we must have $\operatorname{Im} f=S_{i_{0}}$. Therefore $\operatorname{Hom}_{R}\left(S, S_{i_{0}}\right)=\operatorname{Hom}_{R}(S, E)$. Since $R$ is commutative we have $\operatorname{Hom}_{R}(S, E)=\operatorname{Hom}_{R}\left(S, S_{i_{0}}\right) \cong \operatorname{Hom}_{R}(S, S) \cong S$ as $R$ modules. Since $E$ is injective, [3, VI, 5, (2)] yields, for any $R$-module $M$, an isomorphism

$$
\operatorname{Ext}_{R}^{1}\left(M, \operatorname{Hom}_{R}(S, E)\right) \cong \operatorname{Hom}_{R}\left(\operatorname{Tor}_{1}^{R}(M, S), E\right),
$$

i.e. $\operatorname{Ext}_{R}^{1}(M, S) \cong \operatorname{Hom}_{R}\left(\operatorname{Tor}_{1}^{R}(M, S), E\right)$. Therefore $S$ is flat if and only if $\operatorname{Tor}_{1}^{R}(M, S)=0$ if and only if $\operatorname{Hom}_{R}\left(\operatorname{Tor}_{1}^{R}(M, S), E\right)=0$ if and only if $\operatorname{Ext}_{R}^{1}(M, S)$ $=0$ if and only if $S$ is injective.

Proposition 2.5 is now immediate: If $P$ is a regular module then every simple homomorphic image is flat, hence injective.

ReMark. We do not know whether the converse to Proposition 2.5 holds. I.e. if $R$ is a commutative ring and $P$ is a projective module such that every simple homomorphic image of $P$ is injective (flat), is $P$ necessarily regular?

For finitely generated projectives the answer is yes:

Proposition 2.7. For a finitely generated projective module over a commutative ring $R$ the following are equivalent:

(1) $P$ is a regular module.

(2) Every simple homomorphic image of $P$ is injective.

Proof. (1) $\Rightarrow$ (2) follows from Proposition 2.5.

(2) $\Rightarrow(1)$. Let $A=\operatorname{Ann}_{R} P$ and $\bar{R}=R / A$. Then $P$ is a faithful finitely generated projective $\bar{R}$-module and since $R$ is commutative $P$ is a generator for $\bar{R} \mathscr{M}$ by Proposition 1.3. Thus $P$ and $\bar{R}$ have the same simple homomorphic images by Proposition 1.2. But every $\bar{R}$-simple homomorphic image of $P$ is an $R$-simple homomorphic image and by (2) is $R$-injective. Therefore it is $\bar{R}$-injective. Thus $\bar{R}$ is a commutative ring with the property that every simple $\bar{R}$-module is injective. By Kaplansky's result, $\bar{R}$ is a regular ring. Hence $P$ is a regular $\bar{R}$-module. But $P$ has the same structure as an $R$ or $\bar{R}$-module so $P$ is a regular $R$-module.

The following is an immediate consequence of the proof of Proposition 2.7.

COROllary 2.8. Let $R$ be a commutative ring, $P$ a finitely generated projective, $A=\mathrm{Ann}_{R} P$. Then the following statements are equivalent:

(1) $P$ is a regular $R$-module.

(2) $R / A$ is a regular ring. 
COROLLARY 2.9. For a commutative ring $R$ the following statements are equivalent:

(1) $R$ is a regular ring.

(2) $R$ possesses a finitely generated faithful regular module $P$.

Remarks. (1) The finite generation of $P$ is essential in Corollary 2.9.

EXAMPLE 2.10. An example of a commutative ring $R$ which is not regular, but which possesses a faithful regular module.

Let $Q$ be the field of rational numbers, $Z$ the ring of integers and $I$ an infinite index set. For each $i \in I$, let $\boldsymbol{Q}_{i}=\boldsymbol{Q}$ and let $S=\prod_{i \in I} \boldsymbol{Q}_{i}$. Then $S$ is a ring under coordinate-wise operations. Let $P=\sum_{i \in I} \oplus Q_{i}=\left\{\left(a_{i}\right)_{i} \in S \mid a_{i}=0\right.$ for all but a finite number of indices $i$ \} and let $R$ be the subring of $S$ generated by $P$ and $1 \in S$. Then $b=\left(b_{i}\right)_{i} \in R$ iff there exists $n \in Z$ such that $b_{i}=n$ for all but a finite number of indices $i . P$ is an ideal of $R$ and it is easy to see that $R / P \cong Z$ as rings. Thus $R$ has a homomorphic image which is not a regular ring so $R$ is not a regular ring.

For each $i \in I$ let $e_{i}$ be the element of $R$ with 1 in the $i$ th position and 0 elsewhere. Then $e_{i}^{2}=e_{i}$ and $P=\sum_{i \in I} \oplus R e_{i}$, so $P$ is projective. Since $P$ is also semisimple, $P$ is a regular module. Finally, if $a \in R$ and $a P=0$ then $a e_{i}=0$ for all $i$ and hence $a=0$. Therefore $P$ is faithful.

Note that in the foregoing example, $R$ is semiprime and $P=\operatorname{Soc}(R)$. Also observe that $\boldsymbol{Q}$ could be replaced by any field of characteristic 0 .

(2) Corollary 2.9 is not true for arbitrary noncommutative rings.

EXAMPLE 2.11. Let $S$ be the ring of linear transformations of an infinitedimensional vector space $V$ over a field $K$ of characteristic 0 . Let $I$ be the (two-sided) ideal of $S$ consisting of linear transformations of finite rank and let $R$ be the subring of $S$ generated by $I$ and $1 \in S$. Then $R / I \cong Z$, the ring of integers, so $R$ is not a regular ring. Also note that every nonzero two-sided ideal of $R$ contains $I$.

Let $e$ be the projection of $V$ onto a cyclic subspace of $V$ so $P=R e$ is a simple projective $R$-module, hence regular. If $g \in R$ and $g P=0$ then $\operatorname{Rg} R$ is an ideal of $R$ and $R g R e=0$. If $R g R \neq 0$ then $R g R \supset I$ and $e \in I$ whence $e^{2}=0$, which is impossible. Therefore $R g R=0$ so $g=0$ and $P$ is a cyclic faithful regular $R$-module.

With regard to the structure of arbitrary regular modules we have

THEOREM 2.12. Let $R$ be any ring. An $R$-module $P$ is a regular module if and only if $P$ is isomorphic to a direct sum of principal left ideals of $R$, each of which is a regular module.

REMARK. Theorem 2.12 can be regarded as a slight generalization of [6, Theorem 4] and the proof is similar.

Proof of Theorem 2.12. $(\Leftarrow)$ This follows from property (1) of regular modules.

$(\Rightarrow)$ First assume $P$ is finitely generated. We proceed by induction on $n=$ minimal number of elements required to generate $P$ :

$n=1$. Then $P$ is a cyclic projective so $P \cong R e$ where $e^{2}=e \in R$.

Now assume $n>1$ and the theorem is true for regular modules requiring less than $n$ generators. Let $\left\{x_{1}, \ldots, x_{n}\right\}$ be a minimal generating set for $P$. Then 
$P=R x_{1} \oplus Q$ and if $p: P \rightarrow Q$ is the projection onto $Q$ with kernel $R x_{1}$ then $\left\{x_{2} p, \ldots, x_{n} p\right\}$ generates $Q$. Since $Q$ is a regular module the induction assumption implies that $Q \cong R e_{2} \oplus \cdots \oplus R e_{n}$ where $e_{i}^{2}=e_{i} \in R, i=2, \ldots, n$, and each $R e_{i}$ is regular. But $R x_{1}$ is regular so $R x_{1} \cong R e_{1}$ where $e_{1}^{2}=e_{1} \in R$. Therefore

$$
P \cong R e_{1} \oplus \cdots \oplus R e_{n} .
$$

Now assume $P$ is an arbitrary regular module. By [6, Theorem 1] we may assume $P$ is countably generated, say $P=\sum_{i=1}^{\infty} R x_{i}$. For each $n=1,2, \ldots$, let $P_{n}=\sum_{i=1}^{n} R x_{i}$. Then $P=\bigcup_{n=1}^{\infty} P_{n}$ and each $P_{n}$ is a direct summand of $P$, hence a regular module. Since $P_{n} \subset P_{n+1}$ for all $n$ and each $P_{n}$ is finitely generated we can write $P_{n+1}$ $=P_{n} \oplus Q_{n}, Q_{n}$ finitely generated. Then if $Q_{0}=P_{1}$, it is easy to see we have, for each $n=1,2, \ldots$,

$$
P_{n}=\sum_{i=0}^{n-1} \oplus Q_{i}
$$

Thus $P=\sum_{i=0}^{\infty} \oplus Q_{i}$, so $P$ is a direct sum of finitely generated regular modules. But each finitely generated regular module is isomorphic to a direct sum of principal left ideals of $R$.

3. Regular endomorphism rings. In this section we investigate the relationship between regular modules and projective modules having regular endomorphism rings. More specifically we seek answers to the questions:

(I) If $P$ is a regular module, is $\operatorname{Hom}_{R}(P, P)$ a regular ring? And conversely,

(II) If $P$ is a projective module such that $\operatorname{Hom}_{R}(P, P)$ is a regular ring, is $P$ regular?

To answer these questions we need two lemmas:

LEMMA 3.1. Let $R$ be a ring, $M$ an $R$-module, and $S=\operatorname{Hom}_{R}(M, M)$. Then for $f \in S$ the following statements are equivalent:

(1) There exists $g \in S$ such that $f g f=f$.

(2) $\operatorname{Ker} f$ and $\operatorname{Im} f$ are direct summands of $M$.

Proof. (1) $\Rightarrow(2)$. Choose $g \in S$ such that $f g f=f$. Then $\left.g\right|_{\operatorname{Im} f}$ splits the sequence $0 \rightarrow \operatorname{Ker} f \rightarrow M \rightarrow \operatorname{Im} f \rightarrow 0$ and $g f$ splits the sequence $0 \rightarrow \operatorname{Im} f \rightarrow M$.

$(2) \Rightarrow(1)$. Assume (2) holds. Then there exists $g^{\prime}: \operatorname{Im} f \rightarrow M$ such that $y g^{\prime} f=y$ for all $y \in \operatorname{Im} f$, i.e. $x f g^{\prime} f=x f$ for all $x \in M$. But $\operatorname{Im} f$ is a direct summand of $M$ so we can extend $g^{\prime}$ to $g$ on $M$ by taking $g=0$ on the complementary summand. Then for any $x \in M, x f g f=x f$, i.e. $f g f=f$.

COROllary 3.2. Let $R$ be a ring, $M$ an $R$-module, and $S=\operatorname{Hom}_{R}(M, M)$. Then $S$ is a regular ring if and only if for each $f \in S, \operatorname{Ker} f$ and $\operatorname{Im} f$ are direct summands of $M$.

If $M$ is an $R$-module, $N$ a direct summand of $M$, and $e$ the projection of $M$ onto $N$ then it is easy to see that $e$ is an idempotent of $S=\operatorname{Hom}_{R}(M, M)$ and 
$\operatorname{Hom}_{R}(N, N)=e S e$. This fact will be used in the next lemma and later portions of this paper.

LEMMA 3.3. Let $R$ be a ring and suppose $M$ is an $R$-module having a regular endomorphism ring. Then every direct summand of $M$ also has a regular endomorphism ring.

Proof. Let $S=\operatorname{Hom}_{R}(M, M), N$ a direct summand of $M$, and $e$ the projection onto $N$. Then $\operatorname{Hom}_{R}(N, N)=e S e$. But for any regular ring $S$ and any idempotent $e \in S, e S e$ is a regular ring.

REMARK. There are examples of modules $M=M_{1} \oplus M_{2}$ such that both $M_{1}$ and $M_{2}$ have regular endomorphism rings but such that $\operatorname{Hom}_{R}(M, M)$ is not regular. In fact we have the following:

EXAMPLE 3.4. An example of a regular module $P$ which does not have a regular endomorphism ring, but which admits a decomposition $P=P_{1} \oplus P_{2}$ where $P_{1}, P_{2}$ have regular endomorphism rings: Let $K$ be a field and $I$ an infinite index set. For each $i \in I$ let $K_{i}=K$. Let $R=\prod_{i=1}^{\infty} K_{i}$. Then with coordinatewise operations $R$ is a regular ring.

Let $P_{1}=R$ and $P_{2}=\sum_{i \in I} \oplus K_{i} \subset \prod_{i \in I} K_{i}=R$. Then $P_{1}, P_{2}$ are regular $R$-modules and $\operatorname{Hom}_{R}\left(P_{1}, P_{1}\right) \cong R \cong \operatorname{Hom}_{R}\left(P_{2}, P_{2}\right)$ so both have regular endomorphism rings. Let $P=P_{1} \oplus P_{2}$ (external direct sum). Then $P$ is a regular module.

Let $f$ be the endomorphism of $P$ defined by $f:\left(x_{1}, x_{2}\right) \rightarrow\left(x_{2}, 0\right)$. Then $\operatorname{Im} f=\sum_{i \in I} \oplus K_{i} \subset R=P_{1}$. Since $\sum_{i \in I} \oplus K_{i}$ is not an $R$-direct summand of $R$ it cannot be a direct summand of $P=P_{1} \oplus P_{2}$. Therefore $\operatorname{Hom}_{R}(P, P)$ is not a regular ring.

Note that Example 3.4 gives a negative answer to question (I). More generally we have

THEOREM 3.5. Let $R$ be any ring. Then the following statements are equivalent:

(1) $R$ is semisimple with minimum condition.

(2) $R$ possesses an infinitely generated free module $F$ such that $\operatorname{Hom}_{R}(F, F)$ is a regular ring.

Proof. (1) $\Rightarrow(2)$. If $R$ is semisimple with minimum condition then every submodule of every $R$-module is a direct summand so every $R$-module has a regular endomorphism ring by Corollary 3.2.

$(2) \Rightarrow(1)$. Assume $R$ possesses an infinitely generated free module $F$ such that $\operatorname{Hom}_{R}(F, F)$ is regular. Since $R$ is (isomorphic to) a direct summand of $F$, $R \cong \operatorname{Hom}_{R}(R, R)$ is regular by Lemma 3.3.

Since $R$ is regular, $J(R)=0$ and therefore by Bass [1, Theorem P], in order to show $R$ is semisimple with minimum condition, it is enough to show $R$ satisfies the descending chain condition on principal right ideals.

Now any decreasing chain of principal right ideals in $R$ is expressible in the form $\left\{a_{1} \cdots a_{n} R\right\}$ for some sequence $\left\{a_{n}\right\}_{n=1}^{\infty}$ in $R$. Let $F_{1}$ be a countably generated free 
direct summand of $F$ with basis $\left\{x_{n}\right\}_{n=1}^{\infty}$ and let $G$ be the submodule of $F_{1}$ generated by $\left\{x_{n}-a_{n} x_{n+1}\right\}_{n=1}^{\infty}$. Since $F_{1}$ is a direct summand of $F, \operatorname{Hom}_{R}\left(F_{1}, F_{1}\right)$ is also regular, by Lemma 3.3. Since the correspondence $x_{n} \rightarrow x_{n}-a_{n} x_{n+1}$ defines an endomorphism of $F_{1}$ with image $G$, Corollary 3.2 implies that $G$ is a direct summand of $F_{1}$. But then, by [1, Lemma 1.3], the sequence $\left\{a_{1} \cdots a_{n} R\right\}_{n}$ terminates.

Thus if $R$ is a regular ring which does not have minimum condition then every free $R$-module is certainly a regular module but no infinitely generated free module can have a regular endomorphism ring.

However, concerning the first question we do have the following:

THEOREM 3.6. Let $R$ be a ring and $P$ a finitely generated regular $R$-module. Then $S=\operatorname{Hom}_{R}(P, P)$ is a regular ring.

Proof. Let $f \in S$. Then $\operatorname{Im} f$ is finitely generated and hence a direct summand of $P$. Thus $\operatorname{Im} f$ is projective so $\operatorname{Ker} f$ is a direct summand of $P$. Therefore $S$ is regular.

If $R$ is a ring and $n>0$ is an integer, let $M_{n}(R)$ denote the ring of $n \times n$ matrices over $R$.

COROLlaRY 3.7. A ring $R$ is regular if and only if $M_{n}(R)$ is regular.

Proof. If $R$ is regular then $R^{n}$ is a finitely generated regular module so by Theorem 3.6, $M_{n}(R) \cong \operatorname{Hom}_{R}\left(R^{n}, R^{n}\right)$ is a regular ring.

Conversely, if $M_{n}(R) \cong \operatorname{Hom}_{R}\left(R^{n}, R^{n}\right)$ is a regular ring then since $R$ is a direct summand of $R^{n}, R \cong \operatorname{Hom}_{R}(R, R)$ is regular.

We can now show that the projective module $P$ in Example (4), §2, is regular:

To do this it is enough to show each $p_{i} S$ is regular. Let $f=p_{i} g \in p_{i} S$. Since $\operatorname{Im} p_{i}=R x_{i}$ is cyclic, $\operatorname{Im} f$ is cyclic. Therefore $\operatorname{Im} f$ is a direct summand of the regular module $F$ so $\operatorname{Im} f$ is projective. Thus $\operatorname{Ker} f$ is also a direct summand of $F$. Therefore there exists $g \in S$ such that $f=f g f$ and hence $f S$ is a direct summand of the ring $S$. Thus $f S$ is a direct summand of $p_{i} S$, so $p_{i} S$ is regular.

By Theorem 3.5, if $R$ does not have minimum condition then $S$ will not be a regular ring.

With regard to question (II) the answer is also in general negative:

EXAMPLE 3.8. A cyclic projective module $P$ which is not regular but such that $\operatorname{Hom}_{R}(P, P)$ is a field: Let $K$ be a field and $R=\left(\begin{array}{cc}K \\ 0\end{array} \frac{K}{K}\right)$. Let $P=\left(\begin{array}{ll}0 \\ 0\end{array} \frac{K}{K}\right)$. Then $P$ is a cyclic projective $R$-module and $\operatorname{Hom}_{R}(P, P) \cong K$. Since $\left(\begin{array}{ll}0 \\ 0 & K \\ 0\end{array}\right)$ is a cyclic submodule of $P$ which is not a direct summand, $P$ cannot be a regular module.

However, we do have

THEOREM 3.9. Let $R$ be a commutative ring and $P$ a projective $R$-module such that $\operatorname{Hom}_{R}(P, P)$ is a regular ring. Then $P$ is a regular module.

Proof. First assume $P$ is cyclic, say $P \cong R e$ where $e^{2}=e \in R$. Then $e R e \cong \operatorname{Hom}_{R}(P, P)$ is a regular ring and, since $R$ is commutative, $e R e=R e$. Therefore $R e$ is a regular module. 
Now assume $P$ is arbitrary and by the dual basis lemma choose a generating set $\left\{x_{i}\right\}_{i \in I}$ for $P$ and $\left\{f_{i}\right\}_{i \in I} \subset \operatorname{Hom}_{R}(P, R)$ such that for each $x \in P, x f_{i}=0$ for all but a finite number of $i$, and $x=\sum_{i \in I}(x) f_{i} x_{i}$. For each $i \in I$ define $g_{i}: P \rightarrow R x_{i}$ by $(x) g_{i}=(x) f_{i} x_{i}, x \in P$. Then $g_{i}$ is an endomorphism of $P$. Therefore $P g_{i}$ is a direct summand of $P$, hence a direct summand of $R x_{i}$. Thus $P g_{i}$ is cyclic. Moreover, by Lemma 3.3, $P g_{i}$ has a regular endomorphism ring so, by the cyclic case, $P g_{i}$ is a regular module. Therefore the (external) direct sum $\sum_{i \in I} \oplus P g_{i}$ is a regular module. But $P=\sum_{i \in I} P g_{i}$ and we have a natural epimorphism $\sum_{i} \oplus P g_{i} \rightarrow \sum P g_{i} \rightarrow 0$. Thus $P$ is a projective homomorphic image of a regular module, so $P$ is regular.

COROLlaRY 3.10. Suppose $R$ is commutative and $P$ is a finitely generated projective $R$-module. Then the following statements are equivalent:

(1) $P$ is a regular module.

(2) $\operatorname{Hom}_{R}(P, P)$ is a regular ring.

We conclude this section with a result which is essentially a corollary of Theorem 3.6.

Proposition 3.11. Let $R$ be any ring, $P$ an $R$-progenerator, and $S=\operatorname{Hom}_{R}(P, P)$. Then the following statements are equivalent:

(1) $R$ is a regular ring.

(2) ${ }_{R} P$ is a regular module.

(3) $S$ is a regular ring.

(4) $P_{S}$ is a regular module.

Proof. (1) $\Rightarrow(2)$. Obvious.

(2) $\Rightarrow(3) . P$ is finitely generated.

(3) $\Rightarrow$ (4). Since ${ }_{R} P$ is an $R$-progenerator, $P_{S}$ is a projective $S$-module (see [2, Morita I, p. 9]). Since $S$ is regular this implies $P_{S}$ is a regular $S$-module.

(4) $\Rightarrow$ (1). Again by [2, Morita I, p. 9], $R=\operatorname{Hom}_{S}\left(P_{S}, P_{S}\right)$ and $P_{S}$ is a finitely generated regular $S$-module so $R$ is a regular ring.

4. Local modules and local endomorphism rings. By a local ring we mean a ring $R$ whose nonunits form a two-sided ideal.

It is well known that for a ring $R$ the following statements are equivalent:

(1) $R$ is local.

(2) $R$ has a unique maximal left ideal $M$.

(3) Every cyclic $R$-module is indecomposable.

(4) $R$ is semiperfect and 1 is a primitive idempotent.

(5) $R / J(R)$ is a division ring.

(6) For any $x \in R$ either $x$ or $1-x$ is a unit.

As in the case of regular rings, a number of these conditions are purely module theoretic and are dependent only on the fact that ${ }_{R} R$ is a projective $R$-module.

Proposition 4.1. Let $R$ be a ring and $P \neq 0$ a projective $R$-module. Then the following statements are equivalent: 
(1) $P$ has a unique maximal submodule $M$ which contains every proper submodule of $P($ so $M=J(P))$.

(2) Every homomorphic image of $P$ is indecomposable.

(3) $P$ is indecomposable and every homomorphic image of $P$ has a projective cover.

(4) $P$ is a projective cover of each of its nonzero homomorphic images.

(5) $P=P(R / L)$ for some maximal left ideal $L$ of $R$.

(6) $J(P)$ is small in $P$ and $P / J(P)$ is a simple module.

(7) For any $x \in P$ either $x \in J(P)$ or $R x=P$.

(8) $P \cong R e$ where $e^{2}=e \in R$ and $1-e$ is contained in exactly one maximal left ideal of $R$.

REMARKs. (1) Mares, in [8], defines a projective module $P$ to be semiperfect if every homomorphic image of $P$ has a projective cover. Hence (3) can be restated to say $P$ is semiperfect and indecomposable.

(2) The equivalence of conditions (2) and (4) can be regarded as a dualization of the basic properties of indecomposable injective modules.

(3) The equivalence of condition (1), (3), (6) can be found in [8]; specifically (1) $\Rightarrow(3)$ is [8, Theorem 4.1]. However, for the sake of completeness we present the entire proof of Proposition 4.1 here.

(1) $\Rightarrow$ (2). If $P / N$ is decomposable there exist submodules $P_{1}, P_{2}$ of $P$ such that $P=P_{1}+P_{2}, N \supset P_{1} \cap P_{2}$ and $P_{1} \notin N, P_{2} \notin N$. But then $P_{1} \neq P \neq P_{2}$, so by (1), $P_{1}+P_{2} \subset M \neq P$, which contradicts $P=P_{1}+P_{2}$.

(2) $\Rightarrow$ (3). By (2), $P$ is indecomposable. Let $0 \rightarrow N \rightarrow P \rightarrow P / N \rightarrow 0$ be exact, $N \neq P$. We show $N$ is small in $P$. Let $N+K=P$. Then

$$
P / N \cap K=N / N \cap K \oplus K / N \cap K .
$$

Thus by (2), either $N / N \cap K=0$ or $K / N \cap K=0$, i.e. either $N \subset K$ or $K \subset N$. Since $N+K=P$ and $N \neq P$ we must have $N \subset K$ and hence $K=P$.

Thus $P$ is a projective cover of $P / N$, so each homomorphic image of $P$ has a projective cover.

(3) $\Rightarrow$ (4). Let $P \rightarrow A \rightarrow 0$ be exact, $A \neq 0$. By (3), $A$ has a projective cover $P(A)$ and by the uniqueness of the projective cover, $P=P(A) \oplus P^{\prime}$. Since $P$ is indecomposable, $P^{\prime}=0$ and hence $P=P(A)$.

(4) $\Rightarrow(5)$. Since $P$ is projective, $P$ has a simple homomorphic image $S \cong R / L$ where $L$ is a maximal left ideal of $R$. By (4), $P=P(R / L)$.

(5) $\Rightarrow(6)$. Choose $L$ a maximal left ideal of $R$ such that $P \rightarrow R / L$ is a projective cover. Then $M=\operatorname{Ker}(P \rightarrow R / L)$ is simultaneously a maximal submodule and a small submodule of $P$. Therefore $M=J(P)$ so $J(P)$ is small in $P$ and $P / J(P) \cong R / L$ is simple.

(6) $\Rightarrow$ (7). Let $x \in P$. If $x \notin J(P)$ then the maximality of $J(P)$ yields $J(P)+R x=P$. Since $J(P)$ is small, $R x=P$.

(7) $\Rightarrow(8)$. By (7), $P$ is cyclic so $P \cong R e$ where $e^{2}=e \in R$. Now $R e \cong R / R(1-e)$ so if there exists distinct maximal left ideals $L_{1}, L_{2}$ containing $1-e$ then $P \cong R e$ will 
have distinct maximal submodules $M_{1}, M_{2}$. But then, if $x \in M_{1}-M_{2}$ we would have $x \notin J(P)$ and $R x \subset M_{1} \neq P$, contrary to (7).

(8) $\Rightarrow(1) . P \cong R e \cong R / R(1-e)$ and since $1-e$ is contained in a unique maximal left ideal of $R, P$ has a unique maximal submodule. Because $P$ is cyclic this submodule must contain every proper submodule of $P$.

Definition 4.2. We call a projective module which satisfies the equivalent conditions of Proposition 4.1 a local module.

EXAMPLES OF LOCAL MODUles. (1) If $R$ is a local ring then ${ }_{R} R$ is a local $R$ module.

(2) More generally, if $R$ is a semiperfect ring it is known that $R$ admits decomposition $R=R e_{1} \oplus \cdots \oplus R e_{n}$, where the $e_{i}$ are primitive orthogonal idempotents of $R$. Then each $R e_{i}$ is a local $R$-module.

(3) Let $R=\left(\begin{array}{ll}Z & Q \\ 0 & Q\end{array}\right)$. Then since $R / J(R) \cong Z \oplus Q$ (ring direct sum), $R$ is not semiperfect. However, $P=\left(\begin{array}{ll}0 & Q \\ 0 & Q\end{array}\right)$ is a local $R$-module.

(4) Any minimal left ideal in a semiprime ring is a local module.

Remarks. (1) By Proposition 4.1, $R$ possesses a local module if and only if $R$ has a simple left module $S$ having a projective cover. In fact, it is easy to see that there is a one-to-one correspondence between the (isomorphism types of) local $R$-modules and the maximal left ideals $L$ of $R$ such that $R / L$ has a projective cover. This correspondence is given by $L \leftrightarrow P(R / L)$.

(2) In [8], Mares shows that every semiperfect module has a decomposition into a direct sum of local modules which is unique in the sense of the Krull-RemakAzumaya Theorem. She also shows that any finite direct sum of semiperfect modules is again semiperfect so, in particular, a finite direct sum of local modules is semiperfect. Thus a finitely generated projective $P$ is semiperfect if and only if $P=P_{1} \oplus \cdots \oplus P_{n}$ where the $P_{i}$ are local and $n$ is uniquely determined.

With regard to the relation between local modules and projective modules having local endomorphism rings we have

THEOREM 4.2. Let $R$ be a ring and $P \neq 0$ a projective $R$-module. Then the following statements are equivalent:

(1) $P$ is a local module.

(2) $S=\operatorname{Hom}_{R}(P, P)$ is a local ring.

Remark. (1) $\Rightarrow(2)$ is contained in [8, Corollary 4.2]. However, we give a different proof here:

(1) $\Rightarrow(2)$. If $P$ is local then $J(P)$ is maximal in $P$ and small in $P$. Thus by Proposition 1.1, $S / J(S) \cong \operatorname{Hom}_{R}(P / J(P), P / J(P))$. Since $P / J(P)$ is simple, $S / J(S)$ is a division ring, i.e. $S$ is a local ring.

(2) $\Rightarrow(1)$. We need a lemma:

Lemma 4.3. Suppose $P$ is a projective $R$-module such that $\operatorname{Hom}_{R}(P, P)$ is a division ring. Then $P$ is cyclic. 
Proof. Since $P$ is projective, $P$ has a maximal submodule $M$. If $x \notin M$ and $\pi: P \rightarrow P / M$ is natural then $\pi^{\prime}=\left.\pi\right|_{R x}: R x \rightarrow P / M$ is an epimorphism. The projectivity of $P$ now yields a map $\psi \neq 0$, making the diagram

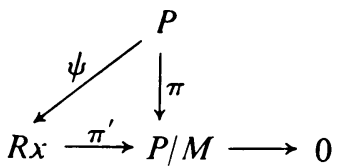

commutative. Since $\operatorname{Hom}_{R}(P, P)$ is a division ring $\psi$ must be an automorphism of $P$ and hence $P=R x$.

Corollary 4.4. Suppose $R$ is a semiprime ring and $P$ is a projective $R$-module such that $\operatorname{Hom}_{R}(P, P)$ is a division ring. Then $P$ is simple.

Proof. By the lemma $P$ is cyclic so $P \cong R e$ where $e^{2}=e \in R$. Then $e R e \cong \operatorname{Hom}_{R}(P, P)$ is a division ring. Since $R$ is semiprime, [7, Lemma 36.3] yields that $R e$ is a minimal left ideal, i.e. $P$ is simple.

Now suppose $P$ is a projective such that $S=\operatorname{Hom}_{R}(P, P)$ is local.

By Proposition 1.1, $J(S) \subset \operatorname{Hom}_{R}(P, J(P))$ and we have a ring epimorphism

$$
S / J(S) \rightarrow \operatorname{Hom}_{R}(P / J(P), P / J(P)) \rightarrow 0 .
$$

Because $S / J(S)$ is a division ring, this map must be an isomorphism so $J(S)$ $=\operatorname{Hom}_{R}(P, J(P))$ and $\operatorname{Hom}_{R}(P / J(P), P / J(P))$ is a division ring. Thus $P / J(P)$ is a projective $R / J(R)$-module having a division ring as its endomorphism ring. But $R / J(R)$ is semiprime, so by Corollary $4.4, P / J(P)$ is a simple $R / J(R)$-module, hence a simple $R$-module. Thus $J(P)$ is the unique maximal submodule of $P$.

To see that $P$ is local, let $x \notin J(P), x \in P$. Let $\pi: P \rightarrow P / J(P)$ be the natural map and let $\pi^{\prime}=\left.\pi\right|_{R x}: R x \rightarrow P / J(P)$. Then $\pi^{\prime}$ is an epimorphism so there is a nonzero map $\psi: P \rightarrow R x$ such that the diagram

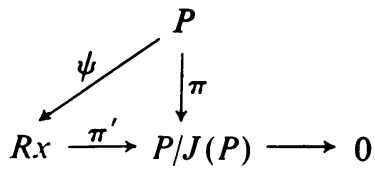

commutes. Then $(x) \psi \pi^{\prime}=(x) \pi \neq 0$ so that $(x) \psi \notin \operatorname{Ker} \pi^{\prime}=R x \cap J(P)$. Therefore $(x) \psi \notin J(P)$, whence $\psi \notin J(S)=\operatorname{Hom}_{R}(P, J(P))$. Since $S$ is local this implies $\psi$ is an automorphism of $P$ and hence $P=R x$. By Propostion 4.1 (7), $P$ is local.

COROLlaRY 4.5. Let $R$ be any ring and $e^{2}=e \in R$. Then $R e$ is a local left $R$-module if and only if $e R$ is a local right $R$-module.

Proof. $\operatorname{Re}$ is local iff $\operatorname{Hom}_{R}(R e, R e) \cong e R e \cong \operatorname{Hom}_{R}(e R, e R)$ is a local ring iff $e R$ is local.

If $R$ is a commutative ring and $M$ is a maximal ideal of $R$ we denote by $R_{M}$ the ring of quotients of $R$ with respect to the set $R \backslash M$. Elements of $R_{M}$ will be written 
$[a / b], a, b \in R, b \notin M$. Then $R_{M}$ is a local ring with unique maximal ideal $M^{\prime}$ $=\{[a / b] \mid a \in M, b \notin M\}$. It is also known that $\operatorname{Hom}_{R}\left(R_{M}, R_{M}\right) \cong R_{M}$, and that as $R$-modules $R_{M} / M^{\prime} \cong R / M$. For these and other facts about $R_{M}$, the reader is referred to [10, Chapter IV, \$\$9-11].

COROllaRY 4.6. Suppose $R$ is commutative and $M$ is a maximal ideal of $R$ such that $R_{M}$ is a projective $R$-module. Then $R_{M}$ is a local module. Hence $R_{M}$ is isomorphic to a direct summand of $R$.

Proof. $\operatorname{Hom}_{R}\left(R_{M}, R_{M}\right) \cong R_{M}$, a local ring, so the projectivity of $R_{M}$ imples $R_{M}$ is a local module.

ReMARKs. (1) There are commutative rings $R$ having maximal ideals $M$ such that the unique maximal ideal of $R_{M}$ does not contain every proper $R$-submodule of $R_{M}$. For example let $R=Z, p$ a prime element of $Z$, and $M=p Z$. Then the maximal ideal of $R_{M}, M^{\prime}=\{a|b| a, b \in Z, a \in p Z, b \notin p Z\}$ does not contain $Z$ which is an $R$-submodule of $R_{M}$. In fact, $R_{M}$ has several distinct maximal $R$-submodules.

(2) We will see later that over a commutative ring $R$ the local modules are exactly the projective $R$-modules of the form $R_{M}, M$ a maximal ideal of $R$.

Sandomierski [9] has shown that a ring $R$ is semiperfect if and only if every simple $R$-module has a projective cover.

COROLLARY 4.7. For a commutative ring $R$ the following statements are equivalent:

(1) $R$ is semiperfect.

(2) For every maximal ideal $M$ of $R, R_{M}$ is $R$-projective.

(3) For every projective $R$-module $P$, and every maximal ideal $M$ of $R, P_{M}$ $=R_{M} \otimes_{R} P$ is $R$-projective.

Proof. (1) $\Rightarrow$ (2). If $R$ is commutative and semiperfect then $R=R_{1} \oplus \cdots \oplus R_{n}$ where $R_{i}$ is a local ring, $i=1, \ldots, n$. Therefore, any maximal ideal $M$ of $R$ has the form $M=M_{i} \oplus \sum_{j \neq i} \oplus R_{j}$ where $M_{i}$ is the unique maximal ideal of $R_{i}$. But then it is easy to see that as $R$-modules $R_{M} \cong\left(R_{i}\right)_{M_{i}}=R_{i}$. Since $R_{i}$ is a direct summand of $R, R_{i}$ is $R$-projective and hence $R_{M}$ is $R$-projective.

(2) $\Rightarrow(3)$. The tensor product of projective modules is always projective.

(3) $\Rightarrow$ (2). $R_{M} \cong R_{M} \otimes_{R} R$ and $R$ is $R$-projective.

(2) $\Rightarrow(1)$. We show every simple module has a projective cover.

Let $S=R / M, M$ a maximal ideal of $R$. Then by (2) and Corollary 4.6, $R_{M}$ is a local module with unique maximal submodule $M^{\prime}$ and $R_{M} / M^{\prime} \cong R / M=S$. But then $R_{M}$ is an $R$-projective cover of $S$.

Condition (1) of Proposition 4.1 states that a projective module $P$ is a local module if $P$ possesses a unique maximal submodule which contains every proper submodule of $P$. It is natural to ask whether the second part of this condition is redundant-namely, if $P$ is a projective module having a unique maximal submodule $M$, does $M$ necessarily contain every proper submodule of $P$ ? While we do not know the answer to this question in general, we can give an affirmative 
answer for several classes of rings. In order to do this we first make some observations:

Let $P$ be a projective module having a unique maximal submodule $M=J(P)$. Then

(1) $P$ is indecomposable.

Proof. A nontrivial decomposition of $P$ would give rise to distinct maximal submodules of $P$.

(2) $P$ is at most countably generated.

Proof. This follows from (1) and [6, Theorem 1].

(3) If $S=\operatorname{Hom}_{R}(P, P)$ and if $J(S)=\operatorname{Hom}_{R}(P, J(P))$ (in particular, if $J(P)$ is small in $P$ ), then $P$ is a local module.

Proof. If $J(S)=\operatorname{Hom}_{R}(P, J(P))$ then $S / J(S) \cong \operatorname{Hom}_{R}(P / J(P), P / J(P))$, which is a division ring. Therefore $S$ is a local ring so $P$ is a local module.

As a consequence of (3) we have

(4) If $P$ is finitely generated then $P$ is local.

(5) $P$ is isomorphic to an ideal of $R$.

To prove (5) a lemma is needed:

LEMMA 4.8. Let $R$ be a ring and $P$ a projective $R$-module. Suppose $P$ has a finitely generated submodule $N$ such that $J(R)(P / N)=P / N$. Then there exists a monomorphism $P \hookrightarrow R^{n}$, for some $n<\infty$. Moreover, we can take $n$ to be the cardinality of a minimal generating set of $N$.

Remark. This lemma is an extension of [1, Proposition 2.7] and its proof is a modification of the proof of Proposition 2.7.

Proof of Lemma 4.8. Note that $J(R)(P / N)=P / N$ if and only if $J(R) P+N=P$. Let $\left\{y_{1}, \ldots, y_{n}\right\}$ be a minimal generating set for $N$. Extend this to a generating set $\left\{y_{i}\right\}_{i \in \Lambda}$ for $P$ by taking $y_{i} \in J(R) P, i \neq 1, \ldots, n$. Now take $F$ free on $\left\{x_{i}\right\}_{i \in \Lambda}$ and map $F \rightarrow P$ via $x_{i} \mapsto y_{i}$. Since this map splits we may assume that $F=P \oplus Q$ and $x_{i}=y_{i}+z_{i}, \quad z_{i} \in Q, \quad i \in \Lambda$. If $i \neq 1, \ldots, n$, then $y_{i} \in J(R) P \subset J(R) F$. Therefore $y_{i}=\sum_{j} a_{i j} x_{j}, a_{i j} \in J(R)$. Thus, for $i \neq 1, \ldots, n$,

$$
z_{i}=x_{i}-y_{i}=\sum_{j}\left(\delta_{i j}-a_{i j}\right) x_{j}
$$

We will show that for $i \neq 1, \ldots, n$, the $z_{i}$ are linearly independent. For, let $z_{i_{1}}, \ldots, z_{i_{m}}$ be any finite set of them. Projecting $F$ onto $R x_{i_{1}} \oplus \cdots \oplus R x_{i_{m}}$, the $z_{i_{1}}$, map onto elements $z_{i_{j}}^{\prime}, j=1, \ldots, m$. We show $z_{i_{1}}^{\prime}, \ldots, z_{i_{m}}^{\prime}$ are linearly independent. Now $z_{i_{j}}^{\prime}=\sum_{k=1}^{m}\left(\delta_{i, k}-a_{i, k}\right) x_{i,}, j=1, \ldots, m$. If $I_{m}$ is the $m \times m$ identity matrix, and $A=\left(a_{i, k}\right), j, k=1, \ldots, m$, then $A \in J\left(M_{m}(R)\right)$ where $M_{m}(R)$ is the ring of $m \times m$ matrices over $R$ and hence $I_{m}-A$ is a unit in $M_{m}(R)$. In particular, its rows are linearly independent. But the rows of $I_{m}-A$ are the coordinate vectors of the $z_{i_{j}}^{\prime}, j=1, \ldots, m$. Hence, $z_{i_{1}}, \ldots, z_{i_{m}}$ are linearly independent. 
We now assert that the projection map $p: F \rightarrow R x_{1} \oplus \cdots \oplus R x_{n}$ maps $P$ monomorphically into $R x_{1} \oplus \cdots \oplus R x_{n}$.

For, let $x=\sum_{i} a_{i} x_{i} \in P$. If $x p=0$ then $a_{1}=a_{2}=\cdots=a_{n}=0$ so $x=\sum_{i \neq 1, \ldots, n} a_{i} x_{i}$. Now project $F$ onto $Q$ with Kernel $P$. Then $0=\sum_{i \neq 1, \ldots, n} a_{i} z_{i}$ and, since the $z_{i}$ are independent for $i \neq 1, \ldots, n$, this implies $a_{i}=0$ for all $i$. Therefore $x=0$.

Now the composite mapping $P \stackrel{p}{\rightarrow} R x_{1} \oplus \cdots \oplus R x_{n} \cong R^{n}$ gives the desired monomorphism.

Proof of (5). Let $x \in P-J(P)$. Then since $J(P)$ is maximal, $J(P)+R x=P$ and since $J(P)=J(R) P$ this gives $J(R)(P / R x)=P / R x$. Lemma 4.8 now yields a monomorphism $P \hookrightarrow R$.

As a corollary to (4) and (5) we have

Proposition 4.9. Suppose $R$ is a left noetherian ring and $P$ is a projective module possessing a unique maximal submodule. Then $P$ is a local module.

With regard to other classes of rings for which $J(P)$ maximal in $P$ implies $P$ is local we have

Proposition 4.10. Suppose $R$ is a ring such that idempotents can be lifted modulo $J(R)$ and suppose $P$ is a projective module such that $J(P)$ is maximal in $P$. Then $P$ is local.

Proof. Let $\bar{R}=R / J(R) . P / J(P)$ is a simple $R$-module and hence a simple projective $\bar{R}$-module. Thus there is an idempotent $\bar{e}$ of $\bar{R}$ such that $P / J(P) \cong \bar{R} \bar{e}$.

Let $\pi: R \rightarrow \bar{R}$ be the natural map. Since idempotents can be lifted modulo $J(R)$ there exists $e^{2}=e \in R$ such that $\pi(e)=\bar{e}$. Let $\pi^{\prime}=\left.\pi\right|_{R e}: R e \rightarrow \bar{R} \bar{e}$. Then $\pi^{\prime}$ is an epimorphism and we assert that $\pi^{\prime}: R e \rightarrow \bar{R} \bar{e}$ is a projective cover of $\bar{R} \bar{e}$. For $\operatorname{Ker} \pi^{\prime}=\operatorname{Re} \cap J(R)$ and since $e^{2}=e, \operatorname{Re} \cap J(R)=J(R) e$. But $R e$ is projective so $J(R e)=J(R) e$. Since $R e$ is cyclic this implies $J(R) e$ is small in $R e$ and hence $R e$ is a projective cover of $\bar{R} \bar{e}$.

Since $P \rightarrow P / J(P) \cong \bar{R} \bar{e}$ is an epimorphism we conclude from the uniqueness of the projective cover that $R e$ is isomorphic to a direct summand of $P$. But $P$ is indecomposable and hence $P \cong R e$. Therefore $P$ is local.

Proposition 4.11. Let $R$ be any commutative ring and $P$ a projective $R$-module with $J(P)$ maximal in $P$. Then $P$ is local. Moreover, if $M$ is the maximal ideal of $R$ such that $P / J(P) \cong R / M$ then $P \cong R_{M}$.

CoROllaRY 4.12. For a projective module $P$ over a commutative ring $R$ the following statements are equivalent:

(1) $P$ is a local module.

(2) $P$ has a unique maximal submodule.

(3) $P \cong R_{M}$ for some maximal ideal $M$ of $R$. 
Hence over a commutative ring $R$ the one-to-one correspondence between the (isomorphism types of) local modules and the maximal ideals $M$ such that $R / M$ has a projective cover can be given by $M \leftrightarrow R_{M}$.

Proof of Proposition 4.11. Let $M$ be the maximal ideal of $R$ such that $P / J(P) \cong R / M$. We assert that, for $b \in R-M$, the mapping $x \mapsto b x$ is an epimorphism of $P$.

The projectivity of ${ }_{R} R$ gives a homomorphism $\psi: R \rightarrow P$ making the diagram

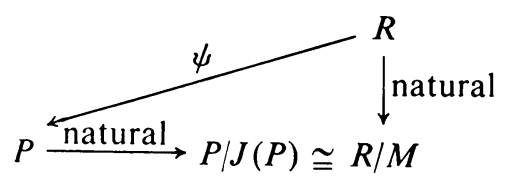

commutative. Since $b \notin M$ the commutativity of this diagram guarantees that $b(1) \psi=(b) \psi \notin J(P)$. Thus $b P \notin J(P)$. Because $J(P)$ is maximal this implies $J(P)+b P$ $=P$. Now $P / b P$ is a projective $R / R b$-module. The Jacobson radical of the ring $R / R b$ can be written $J^{\prime} / R b$ where $J^{\prime}$ is an ideal of $R$ such that $R b \subset J^{\prime} \subset R$ and $J(R) \subset J^{\prime}$. Therefore $J(P)+b P=P \Rightarrow J(R)(P / b P)=P / b P \Rightarrow J^{\prime}(P / b P)=P / b P$, i.e. $\left(J^{\prime} / R b\right)(P / b P)$ $=P / b P$. Since $P / b P$ is a projective $R / R b$-module and $J^{\prime} / R b$ is the Jacobson radical of the ring $R / R b$, Lemma 4.8 (with $n=0$ ) implies that $P / b P=0$. (This is also a direct consequence of [1, Proposition 2.7].) Thus $x \mapsto b x$ is an epimorphism of $P$.

Since $P$ is indecomposable this mapping must be an automorphism of $P$. Therefore, given $b \notin M$ and $y \in P$, there is a unique $x \in P$ such that $b x=y$. Hence $P$ can be made into an $R_{M}$-module in a way that extends the action of $R$.

If $[a / b] \in R_{M}, b \notin M$, and $y \in P$ let $[a / b] \cdot y=$ the unique element $x$ such that $b x=a y$.

Now as an $R_{M}$-module $P$ is also indecomposable and projective so since $R_{M}$ is a local ring $P \cong_{R_{M}} R_{M}$. Therefore $P$ is $R$-isomorphic to $R_{M}$. Corollary 4.6 and the projectivity of $P$ now imply that $P$ is local.

5. Semiperfect and perfect endomorphism rings. In [8], Mares shows that if $P$ is a finitely generated semiperfect module then $\operatorname{Hom}_{R}(P, P)$ is a semiperfect ring. We have the following converse.

Proposition 5.1. Let $R$ be any ring and $P \neq 0$ a projective $R$-module. If $S=\operatorname{Hom}_{R}(P, P)$ is semiperfect then $P$ is a finite direct sum of local modules. Hence $P$ is finitely generated and semiperfect. Moreover, the number of indecomposable summands of $P$ is the same as the number of indecomposable components of $S$. By the Krull-Remak-Azumaya theorem this number is an invariant of $P$ and $S$.

Proof. If $S$ is semiperfect then $S=S e_{1} \oplus \cdots \oplus S e_{n}$ where the $e_{i}$ are primitive orthogonal idempotents of $S$ and $1=e_{1}+\cdots+e_{n}$. Then the $S e_{i}$ are local $S$-modules. Let $P_{i}=P e_{i}$. Then $P=P_{1} \oplus \cdots \oplus P_{n}$. For the sum $P_{1}+\cdots+P_{n}$ is clearly direct and if $x \in P$ then $x=x e_{1}+\cdots+x e_{n}$, so $x \in P_{1}+\cdots+P_{n}$. Since $e_{i}$ is the projection onto $P_{i}$ we have $\operatorname{Hom}_{R}\left(P_{i}, P_{i}\right)=e_{i} S e_{i}$ and since $S e_{i}$ is a local $S$-module

$$
e_{i} S e_{i} \cong \operatorname{Hom}_{S}\left(S e_{i}, S e_{i}\right)
$$


is a local ring. Because each $P_{i}$ is projective this implies $P_{i}$ is a local module, $i=1, \ldots, n$.

Mares, in [8], defines a perfect module to be a projective module $P$ with the property that for every (index) set $I$, every homomorphic image of $P^{(I)}$ has a projective cover. It is clear that a perfect module is semiperfect. Mares shows that if $P$ is a finitely generated (left) perfect module then $\operatorname{Hom}_{R}(S, S)$ is a left perfect ring. Again we have a converse:

Proposition 5.2. Let $R$ be $a$ ring and $P \neq 0$ a projective $R$-module such that $S=\operatorname{Hom}_{R}(P, P)$ is a left perfect ring. Then $P$ is a finitely generated perfect module. In particular, $P=P_{1} \oplus \cdots \oplus P_{n}$ where $\operatorname{Hom}_{R}\left(P_{i}, P_{i}\right)$ is a left perfect local ring, $i=1, \ldots, n$.

The proof of Proposition 5.2 requires a lemma:

Lemma 5.3. Let $R$ be a ring, $P$ a projective module which is a finite direct sum of cyclic modules, and $S=\operatorname{Hom}_{R}(P, P)$. Then the following statements are equivalent:

(1) For any set $I, J\left(P^{(t)}\right)$ is small in $P^{(I)}$.

(2) $J\left(P^{(I)}\right)$ is small in $P^{(I)}$ for some infinite set $I$.

(3) $J(S)$ is left T-nilpotent.

Proof. Clearly $(1) \Rightarrow(2)$.

(2) $\Rightarrow(3)$. In [8, Theorem 7.4], Mares proves local $T$-nilpotence of $J(S)$ and when $P$ is finitely generated, the local $T$-nilpotence of $J(S)$ implies $T$-nilpotence.

(3) $\Rightarrow(1)$. Let $P=P_{1} \oplus \cdots \oplus P_{n}$ where $P_{i}$ is cyclic, and let $e_{i}: P \rightarrow P_{i}$ be the projection onto $P_{i}$. Then $S_{i}=\operatorname{Hom}_{R}\left(P_{i}, P_{i}\right)=e_{i} S e_{i}$ so $J\left(S_{i}\right)=e_{i} J(S) e_{i}$. Since $J(S)$ is left $T$-nilpotent this implies $J\left(S_{i}\right)$ is left $T$-nilpotent, $i=1, \ldots, n$. Now let $I$ be any set. Then $P^{(l)}=P_{1}^{(l)} \oplus \cdots \oplus P_{n}^{(l)}$ and hence $J\left(P^{(l)}\right)=J\left(P_{1}^{(l)}\right) \oplus \cdots \oplus J\left(P_{n}^{(l)}\right)$. Therefore $J\left(P^{(I)}\right)$ is small in $P^{(I)}$ if and only if $J\left(P_{k}^{(I)}\right)$ is small in $P_{k}^{(I)}, k=1, \ldots, n$. Hence to prove the implication (3) $\Rightarrow(1)$ we may assume $P$ is cyclic.

Thus let $P \cong R e, e^{2}=e \in R$. For each $i \in I$ let $\varphi_{i}$ be the composite mapping $R e \cong P \hookrightarrow P_{i} \subset P^{(I)}$. Then the set $\left\{e \varphi_{i} \mid i \in I\right\}$ generates $P^{(I)}$. We now show $J\left(P^{(I)}\right)$ is small in $P^{(I)}$.

Suppose $J\left(P^{(I)}\right)+K=P^{(I)}$. For $x \in P^{(I)}$ let $\bar{x}$ denote its image in $P^{(I)} / K$. If $P^{(I)} / K=0$ we are done. Otherwise, let $0 \neq \bar{x} \in P^{(I)} / K$. Then we can write

$$
\bar{x}=\sum_{i_{1}} a_{i_{1}} \overline{e \varphi_{i_{1}}}, \quad a_{i_{1}} \in J(R) .
$$

For each $i_{1}$ let $b_{i_{1}}=a_{i_{1}} e$. Since

$$
e\left(\overline{e \varphi_{i_{1}}}\right)=\left(\overline{e \cdot e) \varphi_{i_{1}}}={\overline{e \varphi_{i_{1}}}}\right.
$$

we have

$$
\bar{x}=\sum_{i_{1}} b_{i_{1}}{\overline{e \varphi_{i}}}_{i_{1}}, \quad b_{i_{1}} \in J(R) e .
$$


Again, for each $i_{1}$, we have

$$
{\overline{e \varphi_{i}}}_{i_{1}}=\sum_{i_{2}} b_{i_{1} i_{2}}{\overline{e \varphi_{i_{1} i_{2}}}}, \quad b_{i_{1} i_{2}} \in J(R) e .
$$

Continuing in this way we get

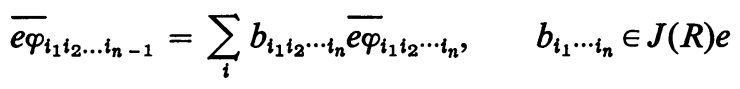

and hence for each $n$

$$
\bar{x}=\sum_{i_{1}, \ldots, i_{n}} b_{i_{1}} b_{i_{1} i_{2}} \cdots b_{i_{1} i_{2} \cdots i_{n}}{\overline{e \varphi_{1}}}_{i_{1} \cdots i_{n}} .
$$

Since $\bar{x} \neq 0$, there is a sequence $i_{10}, \ldots, i_{n 0}$ such that

$$
b_{i_{10}} b_{i_{10} i_{20}} \cdots b_{i_{10} i_{20} \cdots i_{n 0}} \neq 0 .
$$

But then by the König Graph Theorem, there would be a sequence $i_{10}, i_{20}, \ldots, i_{n 0}, \ldots$ such that for all $n$,

$$
b_{i_{10}} b_{i_{10} i_{20}} \cdots b_{i_{10} \cdots i_{n 0}} \neq 0 \text {. }
$$

Rewrite this sequence so $b_{k}=b_{i_{10} \cdots i_{k 0}}$ for all $k$. Then $b_{k} \in J(R) e$ for each $k$ and for all $n, b_{1} b_{2} \cdots b_{n} \neq 0$.

Let $b_{k}=a_{k} e, a_{k} \in R, k=1,2, \ldots$ Then for all $n$

$$
\left(e a_{2} e\right)\left(e a_{3} e\right) \cdots\left(e a_{n} e\right) \neq 0 .
$$

Therefore $e J(R) e$ is not left $T$-nilpotent. But $S=\operatorname{Hom}_{R}(P, P) \cong \operatorname{Hom}_{R}(R e, R e) \cong e R e$ so $J(S)=e J(R) e$ and $J(S)$ is not left $T$-nilpotent, contrary to (3). Therefore $P^{(I)} / K=0$.

REMARK. The construction in the proof that (3) implies (1) is due to Bass [1, pp. 473-474].

The proof of Proposition 5.2 is now easy: Assume $P \neq 0$ is a projective module such that $S=\operatorname{Hom}_{R}(P, P)$ is a left perfect ring. Then by Proposition 5.1, $P$ is a finitely generated semiperfect module so, in particular, $P$ is a finite direct sum of cyclic modules. Since $J(S)$ is left $T$-nilpotent, Lemma 5.3 implies that $J\left(P^{(I)}\right)$ is small in $P^{(I)}$ for all sets $I$. But [8, Theorem 5.2] states that a direct sum $Q=\sum \oplus Q_{i}$ of semiperfect modules $Q_{i}$ is semiperfect if and only if $J(Q)$ is small in $Q$. Hence $P^{(I)}$ is a semiperfect module for any set $I$, i.e. $P$ is perfect.

Note added in proof. It has been brought to the attention of the author that Theorem 3.5 is due to G. M. Tsukerman (Siberian Math. J. 7 (1966), p. 932). Also, Theorem 2.12 has appeared in a recent paper by D. J. Fieldhouse (Math. Ann. 184 (1969), pp. 1-18, Theorem 8.7).

This paper was extracted from the author's doctoral dissertation at the University of California at Santa Barbara, and it was completed during his tenure as a National Science Foundation Trainee. The author would like to express his gratitude for the guidance and inspiration given to him by his thesis advisor, Julius Zelmanowitz.

The author would also like to thank the referee for his valuable suggestions. 


\section{REFERENCES}

1. H. Bass, Finitistic dimension and a homological generalization of semi-primary rings, Trans. Amer. Math Soc. 95 (1960), 466-488. MR 28 \#1212.

2. _- The Morita theorems (Oregon Lectures, 1962).

3. H. Cartan and S. Eilenberg, Homological algebra, Princeton Univ. Press, Princeton, N. J., 1956. MR 17, 1040.

4. S. U. Chase, Direct products of modules, Trans. Amer. Math. Soc. 97 (1960), 457-473. MR 22 \#11017.

5. B. Eckmann and A. Schopf, Uber injektive Moduln, Arch. Math. 4(1953), 75-78. MR 15, 5.

6. I. Kaplansky, Projective modules, Ann. of Math. (2) 68 (1958), 372-377. MR 20 \#6453.

7. _—_, Fields and rings, Univ. of Chicago Press, Chicago, Ill., 1969.

8. E. A. Mares, Semi-perfect modules, Math. Z. 82 (1963), 347-360. MR 28 \#1213.

9. F. L. Sandomierski, On semiperfect and perfect rings, Proc. Amer. Math. Soc. 21 (1969), 205-207. MR 38 \#5848.

10. O. Zariski and P. Samuel, Commutative algebra. Vol. I, University Series in Higher Math., Van Nostrand, Princeton, N. J., 1958. MR 19, 833.

11. A. Rosenberg and D. Zelinsky, Finiteness of the injective hull, Math. Z. 70 (1958/59), 372380. MR 21 \#4176.

UNiversity OF CALIFornia,

Los Angeles, California 90024

NORTHWESTERN UNIVERSITY,

EVANSTON, ILLINOIS 60201 